\title{
Cervical complications of rheumatoid arthritis
}

\author{
M J Kauppi, A Barcelos, J A P da Silva
}

Series editor: Anthony D Woolf

Ann Rheum Dis 2005;64:355-358. doi: 10.1136/ard.2003.020230

\section{CASE HISTORIES \\ Case history 1}

A 47 year old housewife presented with a 3 year history of fleeting inflammatory pain and oedema affecting the small joints of the hands symmetrically. She had previously been given a diagnosis of rheumatoid arthritis (RA), and was receiving treatment with non-steroidal anti-inflammatory drugs (NSAIDs) and prednisolone (10 mg/day).

For the past year she had experienced inflammatory neck pain with a recurrent sense of heaviness and muscle weakness in the upper and lower limbs. Her symptoms were present, especially, during prolonged flexion and were relieved in a favourable posture.

Examination showed symmetrical arthritis and tenderness of wrist and metacarpophalangeal (MCP) joints in both hands. A reduction of about $30 \%$ was seen in the anterior and lateral flexion of the cervical spine. Neurological examination results were, however, normal.

Laboratory tests showed high acute phase response and negative rheumatoid factor. Radiographs of the hands and feet disclosed osteopenia but no erosions. Further investigations included radiographs of the cervical spine (neutral and flexion position) (figs $1 \mathrm{~A}$ and B) and an anterior atlantoaxial subluxation (aAAS) was seen. Magnetic resonance imaging (MRI; fig IC) of the cervical spine showed the aAAS and also space-taking pathological soft tissue (synovitis pannus) around the dens of the axis. The pannus was in contact with the spinal cord, but there was still spinal fluid behind the cord.

The patient was referred to a neurosurgeon and fusion of the $\mathrm{Cl}$ and $\mathrm{C} 2$ spinous processes with the occiput was performed. Further management included active disease modifying drug treatment (methotrexate, up to $20 \mathrm{mg} / \mathrm{week}$ ) and symptomatic treatments.

At her most recent follow up, 10 years after the operation, head movements were reduced but no neurological symptoms were perceived. The Disease Activity Score for a 28 joint count had been between 2.04 and 2.45 .

\section{Case history 2}

A 69 year old housewife with a 10 year history of seropositive, erosive RA treated with NSAIDs, prednisolone ( $10 \mathrm{mg} /$ day), and methotrexate (7.5 mg/week) was evaluated at our department (Coimbra, Portugal) owing to persistent arthritis in both shoulders, elbows, wrists, MCP joints, proximal interphalangeal joints, knees, and metatarsophalangeal joints. She was stiff for about 30 minutes each morning. She denied any neurological symptoms such as occipital headaches, heaviness of the arms, tingling and numbness, muscle weakness, or sphincter dysfunction.

On admission, synovitis was detected in both wrists and knees, MCP joints and proximal interphalangeal joints of the hands, with limited motion of the elbows. Head movements were free and painless. Neurological results were normal. The Disease Activity Score for a 28 joint count (version 3) was 4.85 .
Radiographs of the cervical spine showed a vertical AAS (that is, atlantoaxial impaction (AAI)) of $8 \mathrm{~mm}$ measured by McGregor line (fig 2A). MRI of the cervical spine showed no compression on the spinal cord (fig 2B).

The patient was evaluated by a neurosurgeon, who decided that surgery was not indicated. Two years since this consultation the patient is doing well while using a hard neck collar in risky activities such as travelling.

Her arthritis has been under good control with NSAIDs, prednisolone ( $5 \mathrm{mg} /$ day), and methotrexate ( $15 \mathrm{mg} /$ week).

\section{DISCUSSION}

Involvement of the cervical spine is a highly characteristic component in RA and other chronic inflammatory rheumatic diseases-for example, ankylosing spondylitis, psoriatic arthritis, and juvenile idiopathic arthritis (JIA). ${ }^{1-6}$ The chronic inflammation may injure the stabilising ligaments of the atlantoaxial area. In this case the head pulls the atlas away from the axis at least during flexion of the neck, and aAAS takes place. If the inflammation is chronic in both atlantoaxial facet joints, their cartilage surfaces and also bony structures may be destroyed, and the weight of the skull will press the atlas down around the axis and AAI (often called vertical AAS, although no true subluxation takes place) develops..$^{8}$ Unilateral facet injury may lead to lateral or rotational AAS, but posterior AAS and other abnormalities are also possible. Chronic inflammation in the subaxial area of the cervical spine may lead not only to single or multiple subaxial subluxations (SAS; sometimes due to rheumatoid discitis) but also to subaxial ankylosis (especially in ankylosing spondylitis and JIA). ${ }^{1-8}$ JIA sometimes disturbs the growth of vertebral bodies, leading to typical small or narrow "juvenile cervical vertebrae". ${ }^{5}$

aAAS may develop rather early in RA; its prevalence has been reported to be about $10 \%$ after 2 years of disease and it becomes more common with time if the inflammatory activity continues. ${ }^{9}{ }^{10} \mathrm{AAI}$ is usually a rather late abnormality but is common in severe cases. The prevalence and severity of aAAS may be reduced by the development of AAI, as this process may lead to spontaneous ankylosis. ${ }^{811} 12$ In a Finnish population based series of 98 patents with RA in 1989 (mean disease duration about 17 years) $33 \%$ of the patients presented with aAAS and $27 \%$ had AAI. The origin of SAS may be inflammatory or degenerative, but its prevalence in the Finnish RA cohort was $21 \%$. Different types of cervical spine disorders may coexist in a single patient, and about $50 \%$ of all patients with RA in the study in question presented some rheumatoid cervical involvement. ${ }^{4}$ These figures are probably rapidly decreasing, at least among patients with early RA, because the ability of modern active disease modifying drugs to prevent or retard the development of

Abbreviations: aAAS, anterior atlantoaxial subluxation; AAI, atlantoaxial impaction; DMARDs, disease modifying antirheumatic drugs; JIA, juvenile idiopathic arthritis; MCP, metacarpophalangeal; MRI, magnetic resonance imaging; NSAIDs, non-steroidal antiinflammatory drugs; RA, rheumatoid arthritis; SAS, subaxial subluxation; S-K, Sakaguchi-Kauppi (method) 

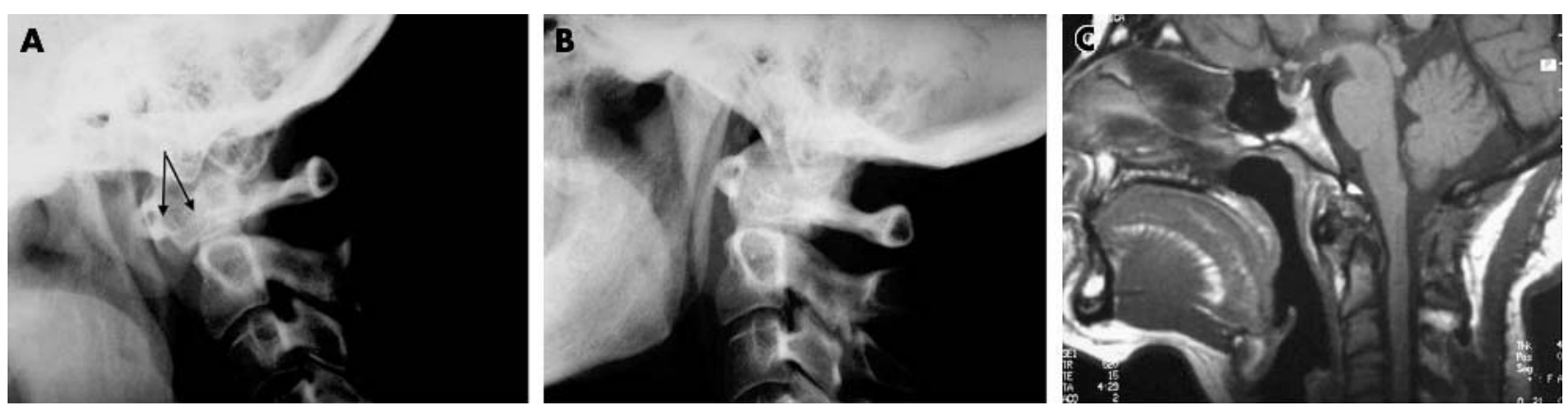

Figure 1 (A) Lateral view radiograph of the cervical spine (case 1) during flexion. Severe AAS is present (arrows show the anterior aspect of the dens of axis and the anterior arch of the atlas). (B) The AAS disappears during neutral posture. (C) Neutral position MRI examination of the cervical spine with the patient in a supine position. AAS is present, together with inflammatory tissue around the dens, but there is no compression of the spinal cord.

atlantoaxial disorders has been shown in a randomised trial. $^{13}$

Neck pain is a common complaint in the general population. Patients with rheumatic cervical spine involvement may have no symptoms, but more often have some neck pain (usually non-specific, sometimes C2 neuralgia) and stiffness. Severe aAAS, AAI, SAS, and some other injuries in the alignment of the cervical spine may cause compression of a critical neural structure-for example, spinal cord and medulla, but also the nerve roots. This may lead to a variety of neurological symptoms, signs and complications-for example, nerve root pain, paresis, tetraplegia, sudden death, hydrocephalus, and brain stroke. ${ }^{1-3} 1214$

Diagnosis of rheumatoid cervical spine involvement is radiological. ${ }^{1-4}$ The most important approach in examination is lateral view plain radiography, taken during full flexion of the neck. It shows most of the subluxations and other abnormalities. About $50 \%$ of unstable aAAS cases would not be diagnosed by neutral position radiographs alone. ${ }^{15}$ Reduction of unstable subluxations may be demonstrated by lateral view radiographs taken in the neutral position and at least during full extension. The shape of the atlantoaxial facet joints may be seen in an open mouth anteroposterior projection. MRI is the best means of assessing active synovitis and possible neural structure compressions in the cervical spine. ${ }^{16}{ }^{17}$ However, dynamic examination by MRI is difficult, making it unreliable for exploring the true extent of subluxation. ${ }^{18}$ MRI is not always available and is still rather expensive and time consuming; it is thus needed only in special cases, especially as a preoperative examination. ${ }^{17}$ Computed tomography may be useful in visualising the bony structures-for example, in atypical subluxations. ${ }^{3}$

Treatment of rheumatoid cervical disorders is usually conservative. ${ }^{1-4} 1319$ The objectives include relief of symptoms and prevention or retardation of the progression of the abnormality. Optimal conservative treatment is multidisciplinary, consisting of patient information, disease activity control (disease modifying antirheumatic drugs (DMARDs), glucocorticoids, and new biological agents), symptomatic treatments (that is, NSAIDs, gentle massage, etc), collars, physical exercises (isometric muscle training, posture practice, etc), and occupational therapies (for example, practical aids and ergonomics). ${ }^{19}$ An operation is needed only if severe symptoms cannot be relieved conservatively, or in the presence of progressive neurological symptoms of cervical origin. Classic "Ranawat scales" are available for evaluating pain and neurological involvement when classifying the clinical situation of the patient. ${ }^{3}$ However, they are not particularly sensitive for modern clinical purposes (a lot of important data-for example, MRI, can now be collected in addition to the scales). Surgery may also be advocated if abnormalities are very severe, leading to a high risk of neurological complications. ${ }^{1-4} 17$ The decision to operate is made individually, as discussed below.
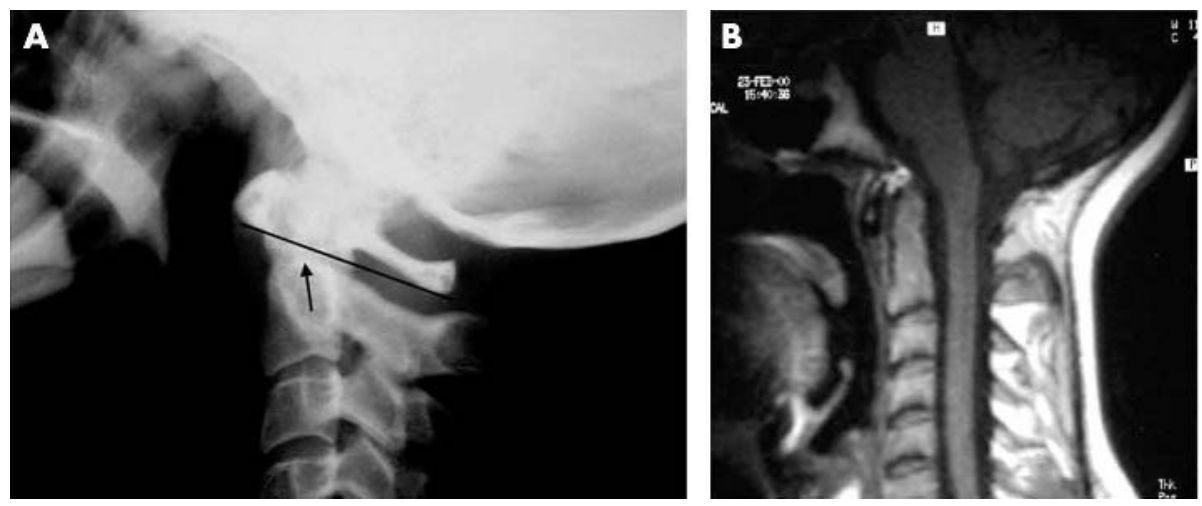

Figure 2 (A) Lateral view radiograph of case 2. No AAl is present according to the Sakaguchi-Kauppi (S-K) method, ${ }^{6}$ which divides the AAI phenomenon into four grades. In lateral view radiographs of the cervical spine, the upper lateral facets of the axis form an easily visible "curve" on the side of the axis (the pedicle). In normal cases (grade I) as in this case, "the cranial tip of this curve" (arrow) is situated caudally from the line, drawn between the most caudal parts of the anterior and posterior arch of the atlas (lower atlas line; drawn in the figure). Owing to erosions of the facets, the atlas may fall down around the axis and the "tip" may reach or penetrate the "lower atlas line" and AAl is then diagnosed (grade II). Grade III means that "the tip of the curve" is on the level (or above) of the line drawn between the central points of the atlas arches. Grade IV means that a very severe AAl is present, with collapsed lateral facets, and the "tip" is on the level (or above) of the line drawn between the most cranial parts of the atlas arches. ${ }^{6}$ (B) MRI of the cervical spine of case 2. The spinal canal is normal and there is no compression of the spinal cord. Active synovitis may be present above the dens of the axis. 


\section{Remarks on case 1}

Patient 1 had RA with high inflammatory activity, which is the cause of the rheumatoid cervical disorders. These are more commonly seen in patients with a rapid destructive course in the peripheral and proximal joints, but she had no erosive changes in her hands or feet. ${ }^{6}{ }^{102021}$ Thus she is not the most typical patient with severe aAAS, but the first permanent rheumatoid changes may be seen also in the neck (personal clinical experience, MJK). Active DMARD treatment may prevent or retard the development of permanent cervical spine changes. ${ }^{13}$

She had neurological symptoms, which appeared to be of cervical origin, but objective neurological findings were not present. It is interesting to note that her symptoms were present especially during prolonged flexion and were relieved during good posture, in agreement with the observation of severe aAAS during flexion, which disappeared in a neutral position. Here the MRI showed the aAAS to be about as severe as seen in the flexion radiographs, which made it easier to evaluate the situation. It is quite common that even severe unstable aAAS is not visible in the MRI examination, taken during flexion, because the patient is in a supine position during the examination. ${ }^{18}$ In that case the maximal extent of aAAS seen in radiographs must be taken into account, while evaluating the possible neural structure compression by MRI.

Preoperative conservative treatment of the cervical spine was not active, because the indication for an operation was assessed to be clear: rather severe unstable aAAS, with neurological symptoms which were construed to be of cervical origin. Modern means of conservative treatment (in addition to active medical treatment) might have relieved the symptoms, giving time for possible development of spontaneous ankylosis and perhaps made the operation unnecessary. ${ }^{13} 19$ However, she was satisfied with the surgical outcome and the neurological symptoms disappeared, because no compression damage developed in the neural structures .

The radiological outcome should be checked occasionally even several years after the operative stabilisation, because a fixed upper neck increases forces upon the lower segments of the cervical spine and may contribute to clinically important SAS. $^{322}$

\section{Remarks on case 2}

This patient had neither cervical nor neurological symptoms. The cervical spine radiographs were taken as a general evaluation of a patient with active RA. She did not have aAAS, but AAI was thought to be present according to the classic McGregor method. ${ }^{23}$ Because of this finding, a neurosurgeon was consulted, but no operation was performed, and the patient is still doing well.

There are several methods available to diagnose AAI (called also vertical AAS), but no one of the classic methods is ideal. The McGregor method used here was designed to diagnose a growth anomaly called basillar impression, but it was later applied to the diagnosis of AAI in rheumatoid patients. ${ }^{23}$ There is one diagnostic method of AAI which is based on the pathogenetic mechanism of the abnormality. It is not disturbed by the individually varying size of the anatomical structures. ${ }^{7}$ This, so-called Sakaguchi-Kauppi method (S-K; defined shortly in the legend to fig $2 \mathrm{~A}$ ) has proved to be useful both in clinical and scientific work..$^{4-8} 13^{14}{ }^{19-21}$ The S-K method has been developed especially for screening purposes, and it can recognise relatively early cases of AAI. According to the S-K method there is no AAI present here (fig 2A). Because there is no validated method of diagnosing AAI by MRI, the diagnosis should be done by plain radiography, and MRI is needed to evaluate the risk of critical neural compression. In this case (fig 2B) we can see that the tip of the dens is rather high, but there is still good space for liquor and neural structures above it. Thus there is no risk of neural complications, no instability, and no need for surgery.

\section{General remarks}

Rheumatoid cervical spine disorders are so common, that cervical spine radiographs should be taken in every patient with RA during the disease course; they all are potential cases of aAAS. ${ }^{3}$ The frequency of the radiography depends on the inflammatory activity and destructivity of the disease and on the possible symptoms of the patient. Patients with highly active disease might be screened, ${ }^{3}$ for example, every third or fourth year even without cervical symptoms.

If cervical spine abnormalities are detected, active conservative treatment should be started. Effective use of DMARDs prevents or retards development of atlantoaxial disorders in early RA and will probably do so also later in the course of the disease. ${ }^{13}$ The new biological agents will probably prove to be even more effective in patients with refractory RA, but scientific evidence is not yet available. These agents may also reduce the size of space taking inflammatory pannus and decrease the possible cord compression, which may influence the need of surgery (for example, in case 1). Osteoporosis may have an important role in the development of severe AAI and other complications, and should be taken into account in the treatment. ${ }^{21} 24$ Other means of active conservative treatment should also be used to help patients with significant cervical disorders. ${ }^{19}$ Their natural course may be led by the treatment towards spontaneous ankylosis, which would reduce the risk of complications. $^{8} 1219$

The progress of diagnosed disorders should be monitored while the conservative treatment is continued. The frequency of the radiographic evaluation depends on the severity of the abnormality, symptoms, and the risk of progress-that is, the systemic inflammatory activity, but may be once in $1-3$ years. MRI may be used for demonstrating inflammatory activity in the cervical spine, ${ }^{16}{ }^{17}$ but this is seldom needed, because inflammatory activity requiring more effective medical treatment is usually present in other joint areas, also.

The aim of surgery is to relieve refractory symptoms and/or to prevent or treat complications, as mentioned above. Many different measures have been proposed as indications for prophylactic operative treatment. ${ }^{1-4172225}$ Each of these indications is supposed to reflect a high risk for neurological complications. These usually include severe AAS (for example, >9-10 mm), severe AAI (for example, grade IV; $\mathrm{S}-\mathrm{K}$ method), less severe combinations of these (for example, AAS >6-10 mm + AAI grade II-IV), or severe SAS (for example, $>4-5 \mathrm{~mm}$ ) and short posterior atlantodental interval $(\leqslant 14 \mathrm{~mm})$ as proposed by Boden et al..$^{25}$ Nowadays it is reasonable to take these as indications for MRI evaluation of the cervical spine. The individual decision to operate or not to operate is based on these data taken together with the symptoms and general situation of the patient. The risk of the complication should be considered higher than the risk of the operation.

Surgical management includes reduction and stabilisation of the injured spine segment, and decompression of the neural structures..$^{2-4} 17222526$ The results of surgery depend on the preoperative situation of the patients and surgical techniques used; information about these is available elsewhere. $^{322526}$ An operation will probably relieve pain effectively, but the neurological complaints will disappear only if no permanent neural tissue damage is already present. ${ }^{26}$ The general risks of surgery-for example, infections and even infrequent postoperative mortality, should always be considered. ${ }^{32}$ There may also be technical or tissue 
problems during the operation. Fusion of one segment will cause increased forces on the other segments and their subluxations have needed operations in $10 \%$ of cases during a 10 year follow up. ${ }^{32}$

\section{THE LESSONS}

- Rheumatoid cervical spine disorders are so common (especially the less severe cases) that only a small percentage of patients need an operation.

- These less severe disorders should not be neglected because they may progress and increase the risk of complication; patients should receive conservative treatment to help them to live with these abnormalities.

- Surgery is needed in severe and refractory cases, and should be carried out before permanent neurological damage has developed.

\section{Authors' affiliations}

M J Kauppi, Rheumatism Foundation Hospital, Pikijarventie 1, FI-18120 Heinola, Finland

A Barcelos, J A P da Silva, Reumatologia, Hospitais da Universidade, 3000-075 Coimbra, Portugal

Correspondence to: Dr M J Kauppi, markku.kauppi@reuma.fi

Accepted 1 September 2004

\section{REFERENCES}

1 Bland JH. Rheumatoid arthritis of the cervical spine. J Rheumatol 1974;1:319-42.

2 Halla JT, Hardin JG Jr, Vitek J, Alarcón GS. Involvement of the cervical spine in rheumatoid arthritis. Arthritis Rheum 1989;32:652-9.

3 Bouchaud-Chabot A, Lioté F. Cervical spine involvement in rheumatoid arthritis. A review. Joint Bone Spine 2002;69:141-54.

4 Kauppi M, Hakala M. Prevalence of cervical spine subluxations and dislocations in a community-based rheumatoid arthritis population. Scand J Rheumatol 1994;23:133-6.

5 Laiho K, Savolainen A, Kautiainen H, Kekki P, Kauppi M. Cervical spine in juvenile chronic arthritis. Spine J 2002;2:89-94.

6 Neva MH, Kaarela K, Kauppi M. Prevalence of the cervical changes in rheumatoid arthritis - a prospective 20-year follow-up study. J Rheumatol 2000;27:90-3.

7 Kauppi M, Sakaguchi M, Konttinen YT, Hämäläinen M. A new method of screening for vertical atlantoaxial dislocation. J Rheumatol 1990;17:167-72.
8 Kauppi $M$, Sakaguchi $M$, Konttinen YT, Hämäläinen $M$, Hakala $M$ Pathogenetic mechanism and prevalence of the stable atlantoaxial subluxation in rheumatoid arthritis. J Rheumatol 1996;23:831-4.

9 Winfield J, Cooke D, Brook AS, Corbett M. A prospective study of the radiological changes in hands, feet and cervical spine in adult rheumatoid disease. Ann Rheum Dis 1981;40:109-14.

10 Paimela L, Laasonen L, Kankaapää E, Leirisalo-Repo M. Progression of cervical spine changes in patients with early rheumatoid arthritis. J Rheumatol 1997;24:1280-4.

11 Matthews JA. Atlanto-axial subluxation in rheumatoid arthritis - a 5-year follow-up study. Ann Rheum Dis 1974;33:526-31.

12 Oda T, Fujiwara K, Yonenobu K, Azuma B, Ochi T. Natural course of cervical spine lesions in rheumatoid arthritis. Spine 1995;20:1128-35.

13 Neva MH, Kauppi MJ, Kautiainen H, Luukkainen R, Hannonen P, LeirisaloRepo $M$, et al. Combination drug therapy retards the development of rheumatoid atlantoaxial subluxations. Arthritis Rheum 2000;43:2397-401.

14 Neva MH, Myllykangas-Luosujärvi R, Kautiainen H, Kauppi M. Mortality associated with cervical spine disorders: a population-based study of 1666 patients with rheumatoid arthritis who died in 1989 in Finland. Rheumatology (Oxford) 2001;40:123-7.

15 Kauppi M, Neva MH. Sensitivity of lateral view cervical spine radiographs taken in neutral position in atlantoaxial subluxation in rheumatic diseases. Clin Rheumatol 1998;17:511-14.

16 Reijnierse M, Dijkmans BA, Hansen B, Pope TL, Kroon HM, Holscher HC, et al. Neurologic dysfunction in patients with rheumatoid arthritis of the cervical spine. Predictive value of clinical, radiographic and MR imaging parameters. Eur Radiol 2001;11:467-73.

17 Dvorak J, Grob D, Baumgartner H, Gschwend N, Grauer W, Larsson S. Functional evaluation of the spinal cord by magnetic resonance imaging in patients with rheumatoid arthritis and instability of upper cervical spine. Spine 1989; 14: 1057-64.

18 Laiho K, Soini I, Kautiainen H, Kauppi M. Can we rely on MRI when evaluating unstable atlantoaxial subluxation? Ann Rheum Dis 2003;62:254-6.

19 Kauppi M, Leppänen L, Heikkilä S, Lahtinen T, Kautiainen H. Active conservative treatment of atlantoaxial subluxation in rheumatoid arthritis. Br J Rheumatol 1998:37:417-20.

20 Neva MH, Isomäki P, Hannonen P, Kauppi M, Krishnan E, Sokka T. Early and extensive erosiveness of peripheral joints predicts atlantoaxial subluxation in patients with rheumatoid arthritis. Arthritis Rheum 2003;48:1808-13.

21 Neva MH, Kotaniemi A, Kaarela K, Lehtinen JT, Belt EA, Kauppi M. Atlantoaxial disorders in rheumatoid arthritis associate with the destruction of peripheral and shoulder joints, and decreased bone mineral density. Clin Exp Rheumatol 2003;21:179-84.

22 Santavirta S, Konttinen YT, Laasonen E, Honkanen V, Antti-Poika I, Kauppi M. Ten-year results of operations for rheumatoid cervical spine disorders. J Bone Joint Surg Br 1991;73:116-20.

23 McGregor $M$. The significance of certain measurements of the skull in the diagnosis of basillar impression. Br J Radiol 1948;21:171-81.

24 Laiho K, Kauppi M. Fracture of spinous process of axis in a patient with rheumatoid arthritis. Clin Rheumatol, 2004 Jul 15; [Epub ahead of print]

25 Boden SD, Dodge LD, Bohlman HH, Rechtine GR. Rheumatoid arthritis of the cervical spine. J Bone Joint Surg Am 1993;73:1282-97.

26 Casey ATH, Crockard HA, Bland JM, Stevens J, Moscovich R, Ransford AO. Surgery on the rheumatoid cervical spine for the non-ambulant myelopathic patients - too much, too late? Lancet 1996;347:1004-7. 